\title{
The effect of moving waves on neutral marine atmospheric boundary layer
}

\author{
Ali Al Samª, Robert Szasz, and Johan Revstedt \\ Lund University, Department of Energy Sciences, SE-221 00 Lund, Sweden
}

\begin{abstract}
Large eddy simulations are performed to study the effects of wind-wave direction misalignment of the neutral marine atmospheric boundary layer over a wavy wall. The results show that the wind-wave misalignment has a significant effect on the velocity profiles and the pressure fluctuation over the wave surface. These effects are not confined to the near wave surface region but extend over the whole atmospheric surface layer.
\end{abstract}

\section{Introduction}

The recent years have shown a dramatic increase in the development of offshore wind energy because of the higher wind speed and less turbulence offshore than on land and fewer environmental constraints. The strong wind over the water surface is often combined with high waves. To improve wind turbine design and to optimize the farm layout with respect to energy production and costs, knowledge of the wind-wave interaction phenomena in the marine atmospheric boundary layer (MABL) is required.

The Monin-Obukhov similarity theory is commonly used to describe the vertical wind velocity profile in horizontally homogeneous atmospheric surface layers, which account for the lowest $10 \%$ of the atmospheric boundary layer (ABL). The similarity theory was derived based on Kansas measurements on land [1] but it is also commonly used to describe the wind shear in the MABL. However, the MABL differs from the ABL on land by the presence of moving ocean waves and wind-wave interactions.

When wind blows over the water surface, the surface is deformed and tiny waves are generated due to the random nature of pressure pulses associated with turbulent flow [2]. These tiny surface deformations amplify the pressure fluctuations over the water surface and, as a result of these interactions, the waves grow exponentially with time [3]. When the wave's energy (and hence its amplitude) increases, part of its energy is transferred by a nonlinear process to waves with smaller frequencies. The wave spectrum with waves at different amplitudes and frequencies develops with time by absorbing momentum from the wind, slowing it down near the water surface.

Over the oceans, the wave spectrum includes a broad range of frequencies with contributions from both locally generated wind waves and swells which are generated remotely by storms. Swells are long waves that can propagate for a relatively long distance with little attenuation $[4,5]$ and may have any direction and velocity, independent of the local wind direction and speed. The presence of swells in the

\footnotetext{
ae-mail: Ali.Al_Sam@energy.lth.se
}

This is an Open Access article distributed under the terms of the Creative Commons Attribution License 2.0, which permits unrestricted use, distribution, and reproduction in any medium, provided the original work is properly cited. 
wave field affects the near surface momentum transfer [6, 7] and alters the wind velocity profile [8]. Under the conditions of low wind and fast swell (i.e. large wave ages, where the wave age is the wave phase speed to wind speed ratio), swell dominates the wave field and causes an upward momentum flux [9]. Swells can cause significant misalignment between winds and wind stress which invalidates the use of the Monin-Obukhov similarity theory $[8,10]$. Large eddy simulation (LES) experiments by Sullivan et al. [11] have shown that in the slow-wind/fast-wave regime swells have impact on the whole ABL.

Swell effects are important not only in the MABL over open oceans but also in small seas and near the coast lines where offshore wind farms are usually installed. Smedman [4] has reported that swells occur as often as $40 \%$ of the time at the Baltic Sea. The measurement of wind, wave and currents at the offshore wind farm Egmond aan Zee [12] in the North Sea shows that the measured average misalignment angle between winds measured at $21 \mathrm{~m}$ height and wave directions is within 40 degrees for unstable atmospheric conditions and larger than that for stable and conditionally unstable conditions even at moderate wind speeds.

The purpose of this study is to investigate the effect of misalignment between wind direction and wave direction on the MABL at moderate wind speed and moderate wave speed conditions which are more common in offshore wind farms than the slow-wind/fast-wave conditions. LES are performed to simulate a neutral MABL over an idealized moving sinusoidal wall for waves aligned with the wind direction and for a 45 degrees misalignment angle between wind and waves. In Section 2, the numerical method and the test cases that are used to verify the used solver are described. The studied waves and the simulated cases are explained in Section 3. The results are discussed in Section 4 followed by the conclusion of this study in Section 5 .

\section{Numerical method}

The buoyantBoussinesqPimpleFoam solver from the OpenFOAM library [13], which is based on the PIMPLE algorithm (merged PISO-SIMPLE) is modified to include the Coriolis and geostrophic wind terms. The pressure and buoyancy terms are treated implicitly in the momentum equation, while the Coriolis and geostrophic wind terms are treated explicitly. In order to avoid the checkerboard pressure oscillation that may occur due to velocity and pressure decoupling in co-located grids, a pseudostaggered grid setup is used by reconstructing the pressure and buoyancy volume fields from face fluxes instead of using the cell center values directly. The Reynolds averaged Navier-Stokes (RANS) model in the solver is replaced by a LES model.

To assess the performance of the modified solver, the results obtained by it are compared with the results of the On/Offshore Wind Farm Applications (SOWFA) software package [15]. SOWFA is an OpenFOAM-based solver for flat terrain simulations and has been developed and tested by the U.S. National Renewable Energy Laboratory (NREL).

Two simulations are performed to simulate a neutral MABL: one with the modified solver and the other using SOWFA. The studied case has a rectangular domain of $(3,3,1) \mathrm{km}$, discretized into $(160 \times 160 \times 128)$ control volumes. The standard Smagorinsky subgrid scale (SGS) model [16], CrankNicholson for time advancement and second-order linear interpolation are used in the simulated cases.

The velocity field is initialized with a logarithmic profile of geostrophic wind blowing from the west at $15 \mathrm{~m} / \mathrm{s}$. The temperature field is initialized with constant $300 \mathrm{~K}$ virtual potential temperature in the first $478 \mathrm{~m}$ of the domain followed by a $62.5 \mathrm{~m}$ thick capping inversion. In the inversion layer the temperature increases by $0.5 \mathrm{~K}$, while it increases slightly above the inversion with a gradient of $3 \mathrm{~K} / \mathrm{km}$.

Periodic boundary conditions are applied in the streamwise and spanwise directions. The specified geostrophic wind at a certain height is maintained in SOWFA through the adjustment of the driving pressure gradient of the momentum equation, while in the modified solver the flow is maintained by applying a constant pressure gradient in the whole domain. The pressure gradient is calculated from the 
geostrophic balance between the Coriolis force and the pressure gradient:

$$
-(1 / \rho)(\partial p) / \partial x=-f V_{g} \quad-(1 / \rho)(\partial p) / \partial y=f U_{g}
$$

where $\left(U_{g}, V_{g}, 0\right)$ is the geostrophic wind vector and $f$ is the Coriolis parameter. $10^{-4}$ is used for the Coriolis parameter for both cases.

At the upper boundary, the velocity is equal to the geostrophic wind and the temperature gradient is $3 \mathrm{~K} / \mathrm{km}$. At the lower boundary, an adiabatic stationary rough wall is used.

In both solvers the surface stress is defined by the Schumann model [14]

$$
\tau /<\tau>=\bar{U}_{z 1} /<\bar{U}_{z 1}>,
$$

where $\tau$ is the surface stress, $\bar{U}_{z 1}$ is the magnitude of the resolved velocity vector at the first cell center and $<$. $>$ denotes horizontal averaging. In the modified OpenFOAM solver the mean wall shear stress $<\tau>$ in the Schumann model is computed from the friction velocity in the logarithmic law expressed as

$$
<\bar{U}_{z 1}>=\left(u_{*} / \kappa\right) \ln \left(z_{1} / z_{o}\right),
$$

where $u_{*}=\sqrt{ }<\tau>$ is the friction velocity, $\kappa$ is the von Karman constant, $z_{1}$ is the first cell center distance from the wall and $z_{o}$ is the aerodynamic roughness height. Then, the SGS viscosity at the lower boundary is calculated from the surface stress and velocity gradient with:

$$
\tau=\vartheta^{S G S}(\partial \bar{U}) / \partial z
$$

An aerodynamic roughness of $1 \mathrm{~mm}$ is given to the lower wall in both cases. The velocity field in the two cases is perturbed in a divergence-free way [17]. The turbulent Prandtl number is taken to be 1/3. The time step is controlled by a fixed maximum Courant-Friedrichs-Lewy (CFL) number equal to 0.3.

The dynamic flow field needs approximately six large eddy turnover times to reach a statistically quasi-steady state [20]. For the neutral atmospheric condition, the large eddy turnover time $\tau^{*}$ is estimated by $\tau^{*}=Z_{i} / u_{*}$, where $Z_{i}$ here is the height of the initial capping inversion. The simulations were computed for ten $\tau^{*}$, here $\tau^{*}$ is estimated to be $1350 \mathrm{~s}$. The flow field is then averaged over the last $2000 \mathrm{~s}$.

The vertical profiles of the horizontally-averaged velocity normalized by the geostrophic wind at the lower part of the MABL are shown in Figure 1. The modified OpenFOAM solver gives lower velocity, and hence lower friction velocity, compared to SOWFA. For example the wind velocity at $10 \mathrm{~m}$ height is $7.5 \mathrm{~m} / \mathrm{s}$ and the friction velocity is $0.325 \mathrm{~m} / \mathrm{s}$, while the corresponding values obtained by SOWFA are $8.3 \mathrm{~m} / \mathrm{s}$ and $0.36 \mathrm{~m} / \mathrm{s}$. These differences between the two solvers might be due to the different SGS stress implementations in both solvers. In the modified OpenFOAM solver, the standard OpenFOAM cell-based SGS stress formulation is used, while in SOWFA the face-based formulation is used. Further the SGS stress in the filtered Navier-Stokes equations is differenced in SOWFA by applying the divergence operator directly to the cell face's velocity gradient [15]. Nevertheless, the test cases show that the modifications applied to the OpenFOAM solver give acceptable results compared to the logarithmic profile and to the SOWFA results.

\section{Wind-wave directions misalignment effect}

To investigate the effect of wind-wave directions misalignment, LES experiments are performed by using the modified OpenFOAM solver. The MABL over a two-dimensional monochromatic moving sinusoidal wave is studied. Two cases are simulated at a moderate wind speed of $10 \mathrm{~m} / \mathrm{s}$ : waves aligned with the wind and 45 degrees wind-wave misalignment. The wind, wave and current measurements at the offshore wind farm Egmond aan Zee [12] in the North Sea show that the likelihood of waves with wave height between $1.2 \mathrm{~m}$ and $2.8 \mathrm{~m}$ is more than $50 \%$ for a wind speed between 9 and $10.5 \mathrm{~m} / \mathrm{s}$. 


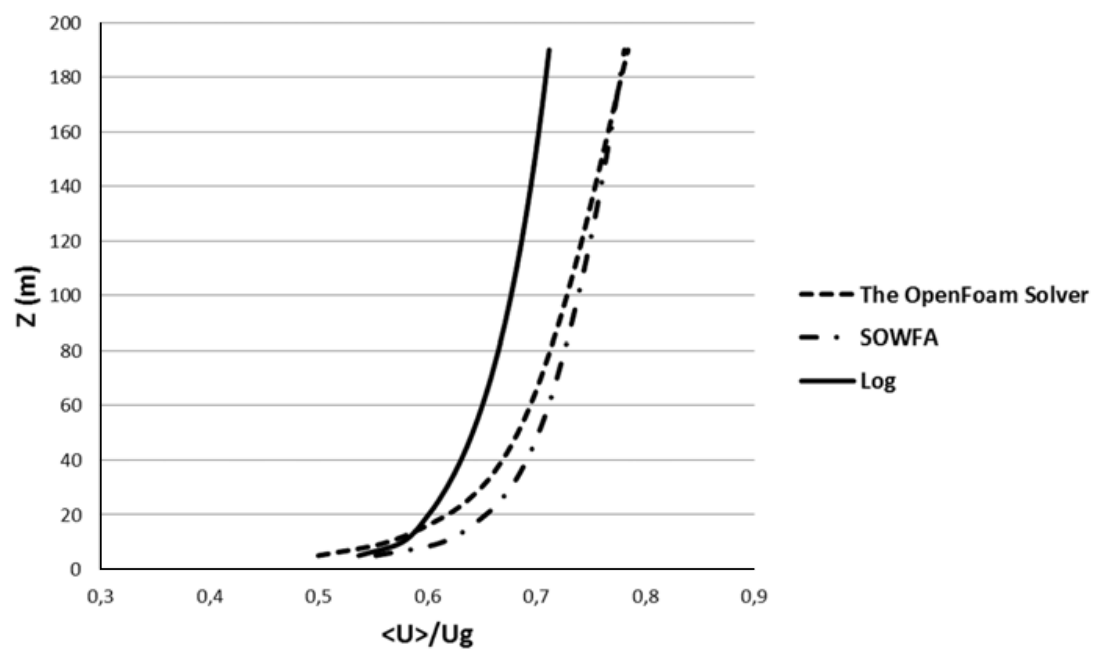

Figure 1. Vertical profiles of wind speed normalized by the geostrophic wind of the modified OpenFOAM solver and SOWFA. Temporal and spatial averaging over $x-y$ planes is applied. The solid line is the logarithmic profile of Equation 3, where $u_{*}=0.35 \mathrm{~m} / \mathrm{s}, \kappa=0.4$ and $z_{o}=1 \mathrm{~mm}$.

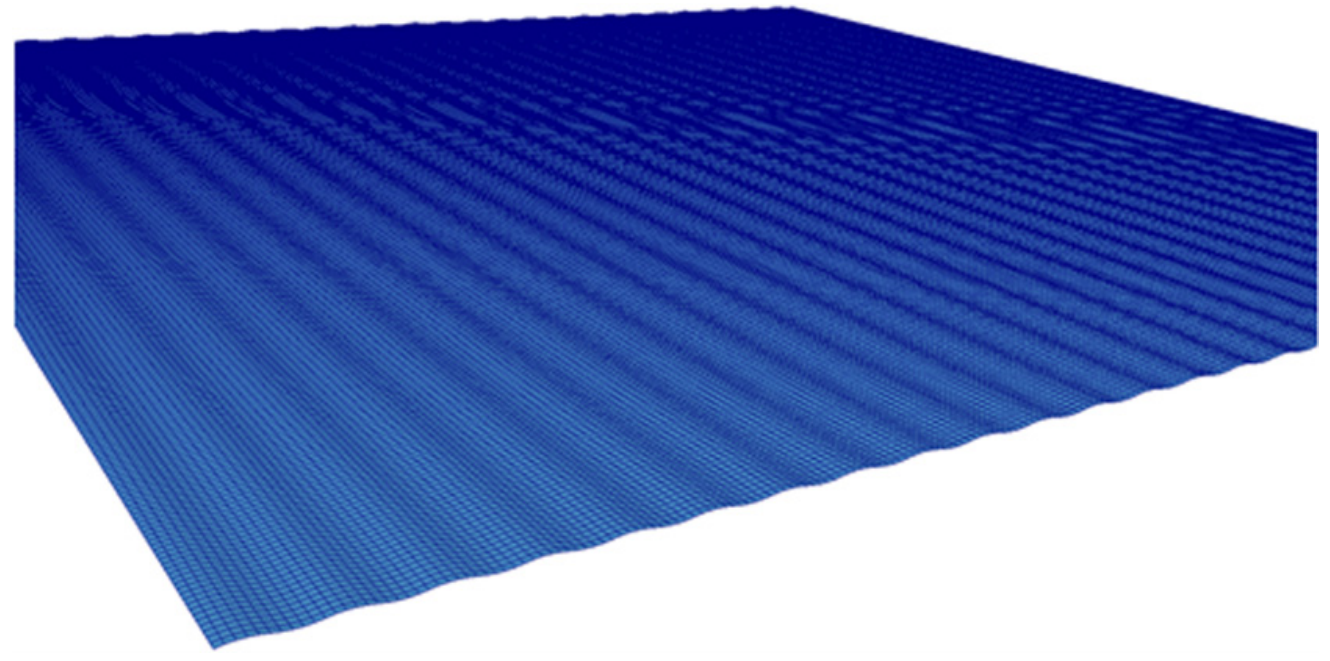

Figure 2. The wavy wall at the lower boundary.

Long period data analysis for different locations in the North Sea shows similar wave data [19]. Figure 2 shows the studied wave chosen to represent a common wave in offshore wind farms. It has wave length $\lambda=40 \mathrm{~m}$, wave height $H_{s}=2 \mathrm{~m}$ (i.e. the wave amplitude $a \approx 1 \mathrm{~m}$ ), wave steepness $2 \pi a / \lambda=0.157$ and wave phase speed $C=\sqrt{ } g \lambda / 2 \pi=7.9 \mathrm{~m} / \mathrm{s}$.

Since the Navier-Stokes equations are Galilean invariant [20], they are solved in a reference frame moving with the wave velocity (i.e. $\boldsymbol{U}-\boldsymbol{C}$ ). The swell direction is held constant aligned with the $\mathrm{x}$-axis in both cases, while the wind velocity direction is changed, see Figure 3.

At low and moderate wind speeds the friction velocity over a rigid wavy surface is larger than its counterpart over an actual water surface where the relative surface stress decreases due to the 


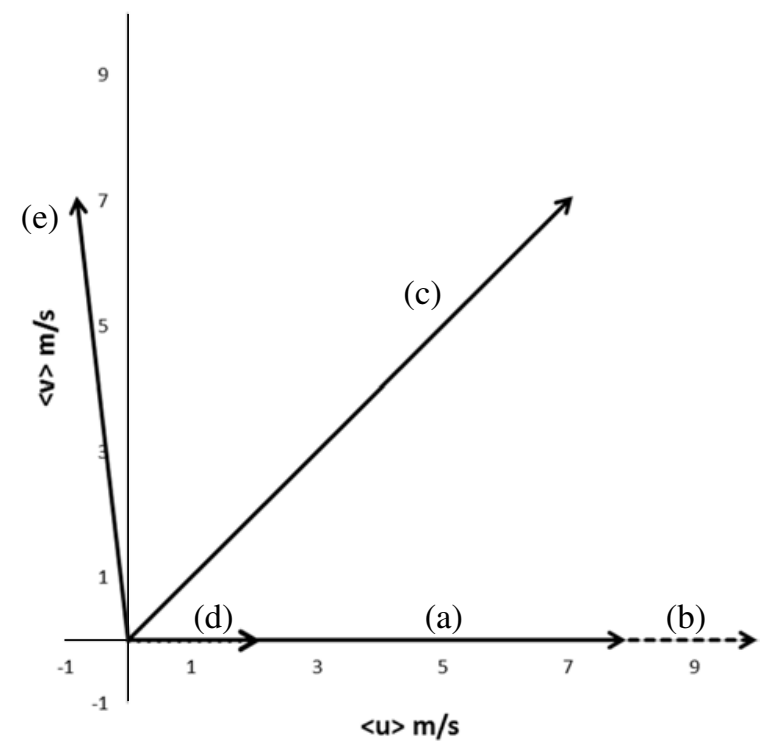

Figure 3. Wind vectors of aligned and misaligned case. (a) Swell; (b) wind aligned with wave and (c) 45 degree wind. (d) and (e) are the geostrophic wind in moving reference frame that move with the swell velocity for aligned and misaligned cases respectively.

development of a drift current. At high wind speeds $\left(U_{10} \cos \theta / C>2.2\right)$ the shear stress caused by the ripples that ride the water wave exceeds the reduction in stress due to the drift current [21]. In the current study the inverse wave age is $\left(U_{10} \cos \theta / C \approx 1.26\right)$ in the case of wave aligned with wind and $\left(U_{10} \cos \theta / C \approx 0.89\right)$ in the misalignment case. Therefore we neglect the viscous drag caused by ripples and only the form drag is resolved by resolving the wave geometry. The Spalding wall law [24] for smooth wall is used.

The studied domain is a rectangular box with $(1.2,1.2,0.8) \mathrm{km}$ dimensions divided into $(300 \times$ $300 \times 96$ ) cells. Each wave length is resolved by 10 points Figure 2 . The OpenFoam utility blockMesh is used to generate a hexahedral mesh. The geometry is sub-divided into 60 blocks, two blocks for each wave, and the cells are stretched in the $z$-direction to reach about $1 \mathrm{~m}$ cell height at the surface. The studied neutrally stratified MABL has a temperature of $300 \mathrm{~K}$ up to the inversion height $Z_{i}=400 \mathrm{~m}$, above this height the temperature increases linearly with $3 \mathrm{~K} / \mathrm{km}$. The cases are simulated for $23000 \mathrm{~s}$ and averaged over the last $3000 \mathrm{~s}$.

\section{Results}

The results of this study show that the coherence and magnitude of pressure fluctuations $\left(p^{\prime} / \rho\right)$ over the wave surface are sensitive to the wind-wave direction misalignment. Previous research [23] on aligned cases has showed that there is a correlation between the $\left(p^{\prime} / \rho\right)$ and the underlying wave surface. This correlation is wave age dependent, where a strong correlation between the positive $\left(p^{\prime} / \rho\right)$ and the wave crests (and similarly correlation between the negative $\left(p^{\prime} / \rho\right)$ and the wave troughs) has been found at high wave age, while at low wave age the correlation is destroyed by the strong turbulence at high wind speed [23].

In our study the same behaviour is observed consistently as in previous studies for the aligned case. At moderate wind speed $(10 \mathrm{~m} / \mathrm{s})$ and low wave age $\left(C p / U_{10} \cos \theta \approx 0.79\right)$ the correlation between the pressure fluctuations with wave surface is weak and extends to about $10 \mathrm{~m}$, see the upper panel of 

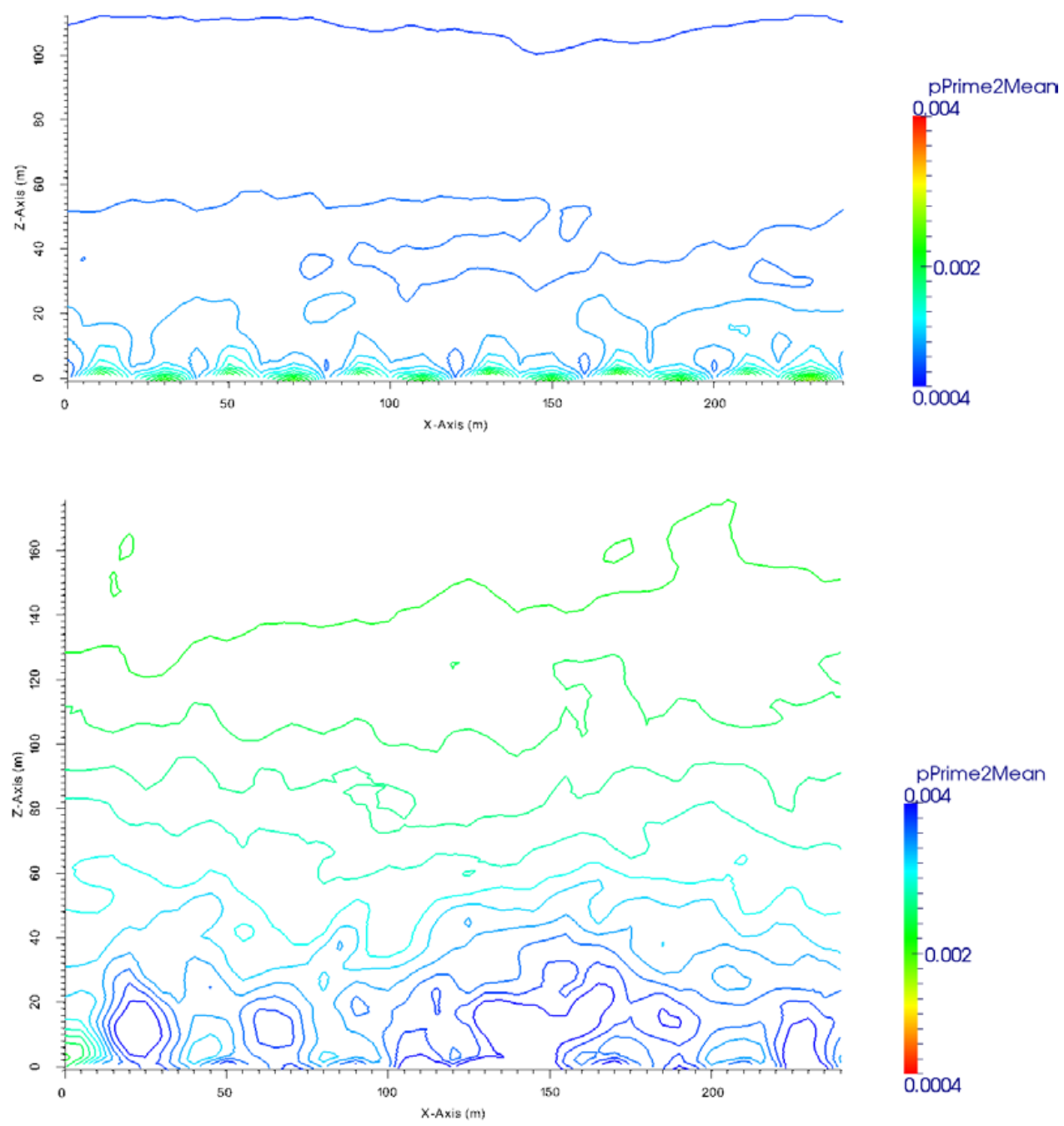

Figure 4. Contour lines of the averaged squared pressure fluctuations in an $x-z$ plane at $y=600 \mathrm{~m}$. The upper panel is wind-wave aligned case, while the lower panel is 45 degree wind-wave misaligned case. Notice the coherence between the pressure fluctuations and underlying wave surface in the aligned case.

Figure 4. Totally different behaviour is observed in the misaligned case. The correlation vanishes for the same wave and wind speed in the misaligned case which has a higher wave age $\left(C p / U_{10} \cos \theta \approx 1,1\right)$, see the lower panel of Figure 4.

This coupling between the wind and the underlying wave in the alignment case can also be seen in the velocity fluctuations. From Figure 5 we can see that there are negative vertical velocity fluctuations riding on the upstream side of the wave's crest and positive fluctuations on downstream of the wave crest in the aligned case. This coupling is weaker in the misaligned case.

Figure 6 shows the impact of wind-wave misalignment on the velocity profiles. These profiles are computed by averaging across a horizontal plane at a constant height and are normalized by the geostrophic wind velocity. The velocity profile of the aligned case consists of two layers: the near surface layer $(z<30 \mathrm{~m})$ in which the wind velocity increases rapidly with height and the near constant wind velocity layer that extend to the top of the boundary layer. This profile is in qualitative agreement 

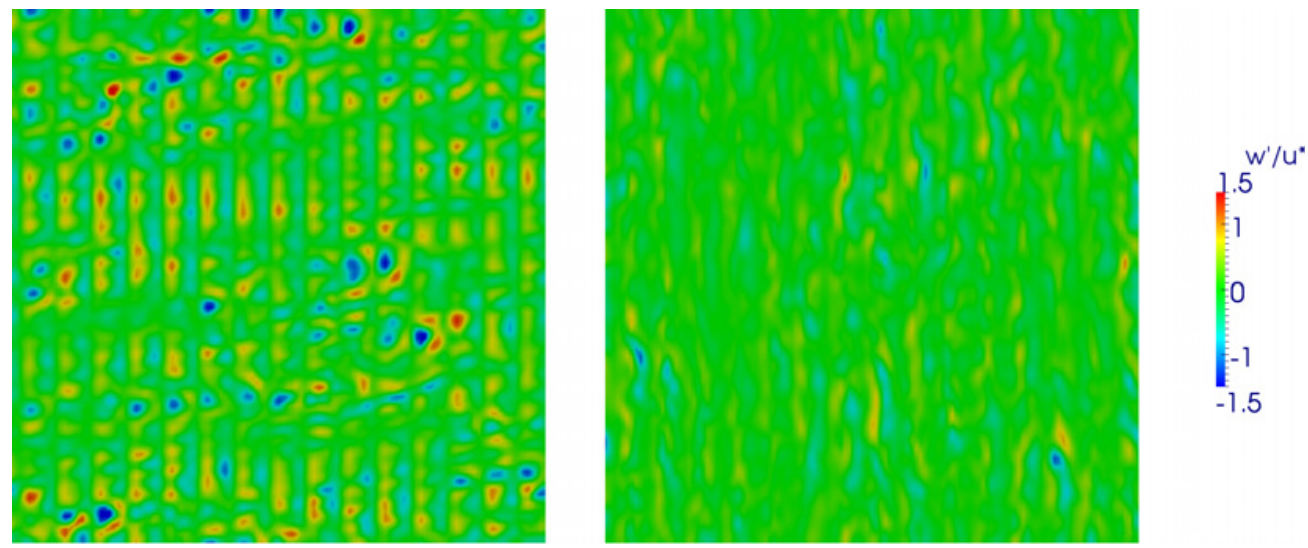

Figure 5. Instantaneous vertical velocity fluctuation normalized by the friction velocity of aligned wind-wave directions (left) and 45 degree wind-wave direction misalignment (right) in an $x-y$ plane at $\mathrm{z}=5 \mathrm{~m}$. The waves propagate from left to right.

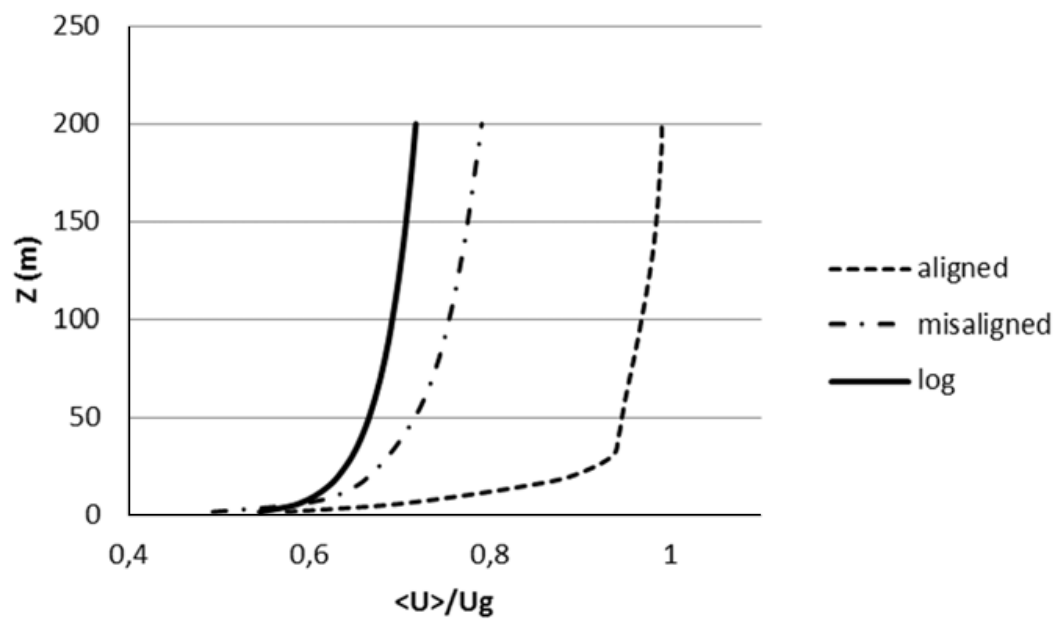

Figure 6. Wind vertical profiles of wind speed normalized by the geostrophic wind for aligned and misaligned wind-wave directions. The solid line is the logarithmic profile of Eq. (3). Temporal and spatial averaging over $x-y$ planes is applied.

with the findings from the measurements during the Baltic Sea Swell Experiments (BASE) [25] which reported an 8 to $10 \mathrm{~m}$ height layer of strong gradient followed by a constant velocity layer. However, the misaligned case has also a strong velocity gradient layer at the lower part of the MABL followed by a smooth positive velocity gradient but the two layer profile is not recognized in this case.

The deviation of the velocity profiles of the simulated cases from the logarithmic profile, see Figure 6, seems to be consistent with other studies [25] which demonstrated that there is no unique relation between the local wind gradient and the momentum flux during swell.

In the ABL far away from the surface there is a geostrophic balance between the pressure gradient and the Coriolis forces, resulting in the geostrophic wind that propagates in a direction normal to these forces. Near the surface the stress vector $(\tau)$ due to turbulence acts as a drag on the surface that reduces the surface wind. The pressure gradient in this case balances the result of the Coriolis and surface friction 


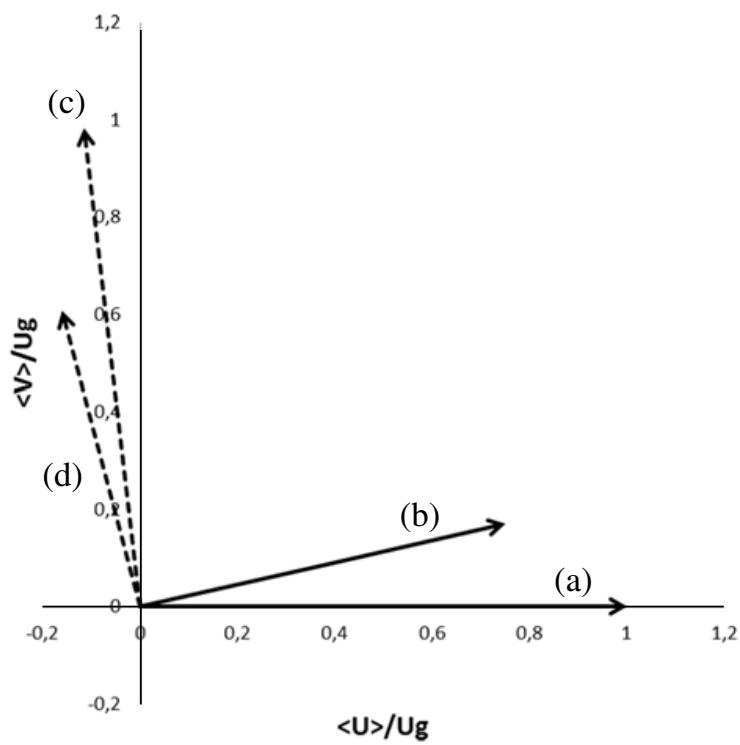

Figure 7. Wind vectors normalized by the geostrophic wind: (a) geostrophic wind of aligned case; (b) wind at $10 \mathrm{~m}$ for aligned case; (c) geostrophic wind for 45 degree misalignment case and (d) wind at $10 \mathrm{~m}$ for misaligned case. Note that these vectors are on a reference frame that moves with swell speed.

forces. The result of this study shows that the forces balance in the aligned case turns the flow about 13 degrees to the left of the geostrophic wind direction. While, in the misaligned case the flow is turned about 8 degrees to the left of its geostrophic wind, see Figure 7.

The Monin-Obukhov similarity theory assumes that the direction of the stress vector aligns with the wind direction. However, when swell is present the total stress vector has a magnitude and direction which is in general a blend between the turbulence stress and swell-induced stress [26]. At high wave age the swell-induced stress dominates the turbulence stress and as a result the stress vector may deviate widely from the mean wind flow direction, including cases when stress is directed across or even opposite to the wind, which invalidates the use of Monin-Obukhov similarity theory. Such a deviation is expected to occur at a higher wave age than in the current study, therefore further investigations need to be done before drawing any conclusions regarding the validity of the Monin-Obukhov similarity theory at moderate wave age.

\section{Conclusions}

Large eddy simulations (LES) are carried out to study the wind-wave direction misalignment effects on neutral marine atmospheric boundary layer (MABL) at moderate wind speed and near wind-wave equilibrium regime by using OpenFOAM.

The current study results show that in the case of swell aligned with the mean wind the velocity profile of the MABL is in general agreement with the field measurements during swell. Further, the results show that wind-wave direction misalignment has a significant impact on the velocity profiles and on the coherence and magnitude of pressure fluctuation in the entire atmospheric surface layer. However, the result of this study is in contrast with the field measurement that demonstrated parameter insensitivity to wind-wave misalignment at lower wave height and lower wave slope $(H s \approx 0.25 \mathrm{~m}$ to $0.6 \mathrm{~m}$ and $a k \approx 0.02$ to 0.03 ) [25] than the corresponding values in our simulations ( $H s=2 \mathrm{~m}$ and $a k=0.157)$. 
The present work indicates that continued studies should include investigations of the flow sensitivity to wind-wave misalignment considering swell at several wave ages, heights and slopes. Furthermore, a study exploring the Monin-Obukhov theory during swell passage for several swell conditions must be performed.

\section{References}

[1] T. Foken, Bound.-Layer Meteor. 119, 431-447 (2006)

[2] O.M. Phillips, J. Fluid Mech. 2, 417-445 (1957)

[3] J.W. Miles, J. Fluid Mech. 3, 185-204 (1957)

[4] A.S. Smedman, U. Högström and E. Sahlee, J. Atmos. Sci. 66, 2747-2763 (2009)

[5] P.P. Sullivan and J.C. McWilliams, J. Annu. Rev. Fluid Mech. 42, 19-42 (2010)

[6] F.W. Dobson, S.D. Smith and R.J. Anderson, Atmos.-Ocean. 32, 236-237 (1994)

[7] M.A. Donelan, W.M. Drennan and K.B. Katsaros, J. Phys. Oceanogr. 27, 2087-2099 (1997)

[8] A.S. Smedman, U. Högström, H. Bergström, A. Rutgersson, K.K. Kahma and H. Pettersson, J. Geophys. Res. 104, 25833-25851 (1999)

[9] A.A. Grachev, and C.W. Fairall, J. Phys. Oceangr. 31, 1698-1711 (2001)

[10] A.S. Smedman, X.G. Larsén, U. Högström, K.K. Kahma and H. Pettersson, J. Geophys. Res. 108, 3367 (2003)

[11] P.P. Sullivan, J.B. Edson, T. Hristov and J.C. McWilliams, J. Atmos. Sci. 65, 1225-1245 (2008)

[12] H. Korterink, P.J. Eecen and J.W. Wagenaar, Meteorological Measurements OWEZ. Half year report, ECN-E-09-018 (2008)

[13] OpenFoam- The open source CFD toolbox, Programmer's guide, version 1.6. OpenCFD ltd.,Albert Road, Caversham, Reading, Berkshire Rg47An, Uk

[14] U. Schumann, J. Comput. Phys. 18, 376-404 (1975)

[15] M. J. Churchfield, 6th OpenFOAM Workshop 13-16 june 2011, The Pennsylvania State University, state College, PA (2011)

[16] J. Smagorinsky, Mon. Wea. Rev. 91, 99-164 (1963)

[17] http://www.openfoamworkshop.org/6th_OpenFOAM_Workshop_2011/Program/Training/ churchfield_slides.pdf

[18] M.J. Churchfield, P.J. Moriarty, G. Vijayakumer and J.G. Brasseur, 19th symposium on Boundary Layers and Turbulence keystone, Colorado, 1B.6 (2010)

[19] C.H. Moeng and P. Sullivan, J. Atmos. Sci, 14, 2052-2062 (1994)

[20] C. Beels, J.C.C. Henriques, J. De Rouck, M.T. Pontes, G. De Backer and H. Verhaeghe, Wave energy resource in the north sea,7th European Wave and Tidal Energy Conference, Porto (2007)

[21] S.B. Pope, Turbulent Flows, 6th (2000)

[22] Y.A. Papadimitrakis, E.Y. Hsu and R. Street, J. Phys. Oceanogr. 14, 1937-1948 (1984)

[23] P.P. Sullivan, J.C. McWilliams and T. Hristov, 19th Conference on Boundary layer and Turbulence, Keystone, 4B.4 (2010)

[24] D.B. Spalding, J. Appl. Mech. 28, 455-458 (1961)

[25] A. Smedman, U. Högström and E. Sahlee, J. Atmos. Sci. 66, 2747-2763 (2009)

[26] A.A. Grachev, C.W. Fairall, J.E. Hare and J.B. Edson, J. Phys. Oceanogr. 33, 2408-2429 (2003) 